\title{
Natural history of histologically moderate cervical dysplasia in adolescent and young women
}

This article was published in the following Dove Press journal:

OncoTargets and Therapy

13 November 2014

Number of times this article has been viewed

\section{Salvador Piris \\ Victoria Bravo \\ Carmen Álvarez \\ Leticia Muñoz-Hernando \\ Estela Lorenzo-Hernando \\ Reyes Oliver \\ Laura Marqueta-Marques \\ Jose M Seoane-Ruiz \\ Alvaro Tejerizo-Garcia \\ Jesús $S$ Jiménez \\ Gynecologic Oncology Service, Hospital 12 de Octubre, Madrid, Spain}

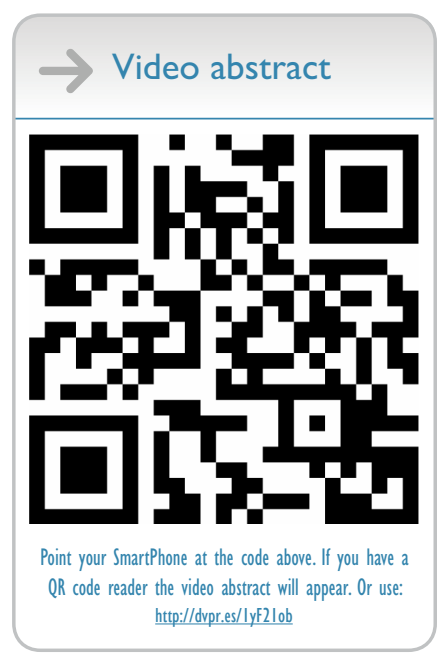

Correspondence: Jesús S Jiménez Gynecologic Oncology Service, Hospital 12 de Octubre,

Avda Cordoba s/n, Madrid 2804I, Spain

Tel/Fax +34 9I 3908376

Email jjimenez.hdoc@salud.madrid.org
Background: Adolescent women are a special age group affected by human papilloma virus (HPV). Most guidelines recommend surgical treatment for high-grade cytological lesions. However, some reports have attempted to demonstrate that the immune system is fully capable of clearing the virus without using conization. Our aim in this study was to describe the outcome of women $<25$ years old with high-grade cytology pap smears and no histologically confirmed cervical intraepithelial neoplasm [CIN] III

Methods: This prospective cohort study, carried out at the Department of Obstetrics and Gynecology, Hospital 12 de Octubre, included 29 women aged 25 years or younger with highgrade cytological lesions recruited in screening programs and were followed up at 15 months. This study describes the clinical course of the women, and we determined the percentage of cytological, histological, and microbiological lesions produced by HPV that were cleared without surgical treatment in these women during their follow-up.

Results: During follow-up, $63 \%$ of high-grade cytological lesions and all high-grade histological lesions were cleared. HPV was eliminated from $23 \%$ of patients with one HPV serotype and $27 \%$ with multiple HPV serotypes without any treatment.

Conclusion: These results suggest that there is no need to use surgical treatment to clear highgrade cytological and cervical lesions in adolescent women, thus preventing damage to their reproductive future.

Keywords: human papilloma virus, cervical intraepithelial neoplasia, conservative management

\section{Introduction}

Infection with human papilloma virus (HPV) is considered the most common sexually transmitted disease. Adolescents are particularly at risk of infection because of the special characteristics of their cervical epithelium, which facilitates the infection, and their sexual practices that may leave up to $70 \%$ of them infected with HPV. A recent meta-analysis of HPV throughout the world showed that the prevalence peaks at $23 \%$ in women younger than 21 years. ${ }^{1}$

Especially in this age group, most of these infections are transient, lasting only a few months. Natural history studies show that both HPV and low-grade squamous intraepithelial lesions (LSILs) are primarily transient in adolescents and young women, with more than $90 \%$ clearing the infection and $90 \%$ showing regression to normal within 3 years. ${ }^{2,3}$ Therefore, the recommended approach to cytological abnormalities in this group of women is observation without treatment to avoid over-diagnosis and over-treatment. ${ }^{4}$ 
A problem arises when a high-grade lesion is found. In adult women, the standard treatment for these lesions is surgery by conization, but this technique is an invasive procedure that can have adverse reproductive outcomes. A recent meta-analysis of cervical excisional procedures showed an increased risk of preterm delivery, low birth weight, and premature rupture of membranes. ${ }^{5}$

Through recent knowledge about the natural history of HPV infection, a change in the management of high-grade lesions in young women in favor of conservative treatment is being seen, which has already been endorsed by the American Association of Cervical Pathology following the publication of its latest clinical guidelines in $2006 .^{6}$

The aim of this study was to observe the clinical course of high-grade cervical lesions in women aged 25 years or younger and confirm the efficacy of conservative management in preventing progression to invasive carcinoma. The main objective of the project is to provide an alternative follow-up for adolescents and young women with cytological results showing high-grade lesions, which are caused by transient HPV infections in a high percentage of cases, to avoid excisional surgical treatments that can seriously compromise their future reproductive potential.

\section{Materials and methods}

This was a prospective observational study performed in the Hospital 12 de Octubre in Madrid from February 2009 to May 2010. This research proposal was approved by the ethics committee of our hospital, and informed consent designed for this study was obtained at the beginning of the study. We included all patients aged 25 years or younger who had a diagnosis in the cervical cytology of high-grade squamous intraepithelial lesion (HSIL) at the screening program. A total of 29 patients were included. The follow-up was 15 months.

These patients presented at the Cervical Pathology Unit of the Hospital 12 de Octubre where they received a colposcopy, cervical biopsy, and endocervical microcurettage, and the samples were tested for HPV-DNA by polymerase chain reaction $(\mathrm{PCR})$.

\section{Procedure}

After studying the pathological and biological lesions, patients were informed of their outcome. We considered whether there was an infection by a high risk HPV and whether the patient was infected with one or several HPV serotypes. Informed consent was required before the procedure. The study was managed as follows:
1. If the histological diagnosis of the lesion was low-grade or less, cytology and colposcopy with endocervical microcurettage were done every 6 months for 2 years if colposcopy was satisfactory and microcurettage was negative.

2. If the histological diagnosis of the lesion was high-grade (cervical intraepithelial neoplasm [CIN] II), cytology and colposcopy with endocervical microcurettage was conducted at 3 and 6 months, then every 6 months for 24 months if the colposcopy was satisfactory and negative microcurettage.

3. If the diagnosis was histologically high-grade lesion type CIN III, excisional treatment was carried out (conization).

4. In all cases, regardless of histological results, an HPV test was performed every 12 months. The patient was treated according to the screening protocol if HPV-DNA negative or at low risk.

\section{Method of analysis}

We collected data on variables and included them in an Excel database. Statistical analysis was performed with SPSS, version 15.0.

We used the chi-square test to assess the significance of the association between two categorical variables. Variables were calculated with the Fisher Student's $t$-test. Calculation of the sensitivity, specificity, and positive predictive value of cytology association - HPV test (PCR) in relation to histological findings. Statistical significance was considered as $P<0.05$.

\section{Results}

\section{Regarding risk factors}

The study population consisted of $40.7 \%$ smokers and $59.3 \%$ nonsmokers. The mean cigarettes per day was 9 (range: 1

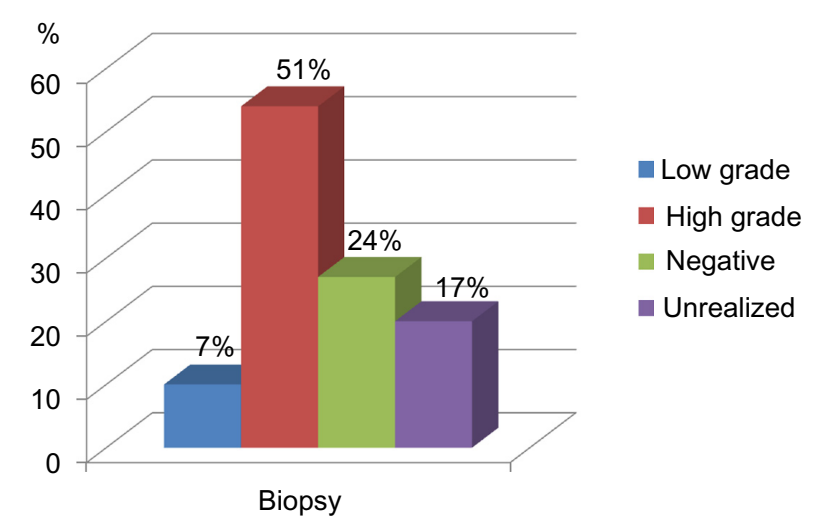

Figure I Histological cervical biopsy results. 


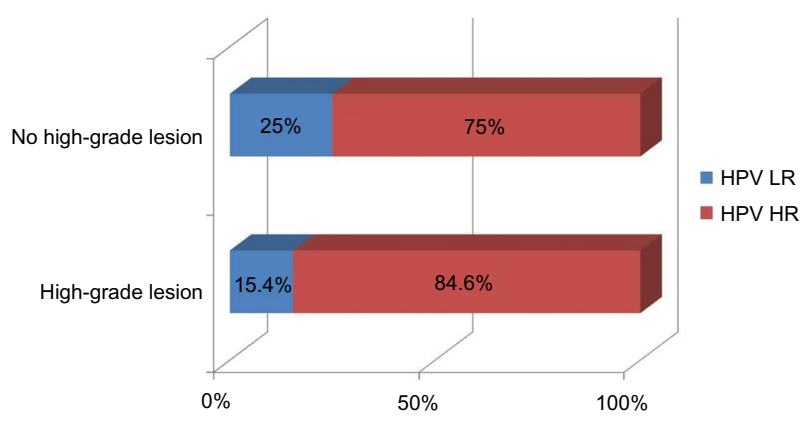

Figure 2 Type of viruses present in cervical biopsy.

Abbreviations: HPV LR, low-risk human papilloma virus; HPV HR, high-risk human papilloma virus.

to 25). The average duration of smoking was 76 months (standard deviation [SD], 51.2 months; range: 12 to 192 months).

Oral contraceptives were taken by $59.3 \%$ at some time, whereas $40.7 \%$ had not. Of those who received oral contraceptives, $50 \%$ still received this factor, with an average exposure time of 39 months (SD, 32.2; range: 3 to 132 months). Of those who did not currently use hormonal contraception, $50 \%$ had a mean hormone-free period of 28 months after the last dose (range: 3 to 48 months).

A stable partner at the present time was reported by $70.4 \%$, and $29.6 \%$ reported no stable partner at the present time. The average age of first sexual relations was 17 years (SD, 2.4 years; range: 12 to 22 years). The average number of partners in the past 3 years was 2.5 (SD, 2.5; range: 1 to 10 ).

Only one patient had microbiological tests positive for sexually transmitted disease. No patient had any type of immunosuppression.

\section{Correlations between cytohistological and microbiological- histological testing}

All patients in the study had high-grade cytological lesions. However, upon biopsy, only 52\% had high-grade lesions (Figure 1). Although not reaching statistical significance, the correlation between the microbiology and the histology was similar $(P=0.5)$ (Figure 2$)$, and we noted that there was a higher percentage of multiple HPV serotypes in high-grade histological lesions $(P=0.09)$ (Figure 3$)$.

\section{Monitoring the natural history}

We studied the evolution of the cytological (Figure 4; Table 1), histological (Figure 5; Table 2), and microbiological (Table 3) characteristics of the lesions during the follow-up, noting that most of the lesions disappeared without any medical or surgical treatment.

\section{Monitoring the behavior of CIN II}

Studying the behavior of type CIN II histological lesions, we noted that all of these lesions disappeared at 9 months of follow-up without using conization in young girls (Figure 6).

\section{Discussion}

HPV infection is a necessary but not sufficient condition for the development of cervical cancer. However, knowledge of the natural history of the infection shows us that in almost $90 \%$ of cases, the infection is transient, and only $10 \%$ of infections persist.

Adolescent girls and young women are at particular risk of infection. Certainly, the reproducibility of HSIL is

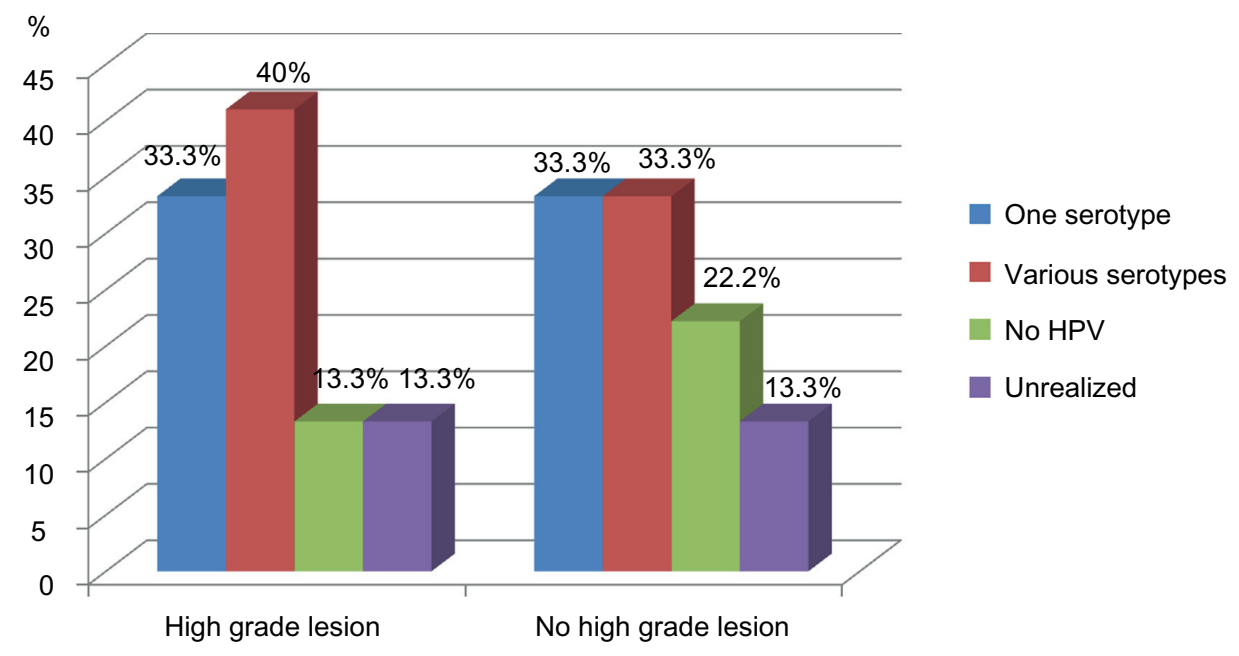

Figure 3 Number of HPV serotypes present in cervical biopsy. Abbreviation: HPV, human papilloma virus. 


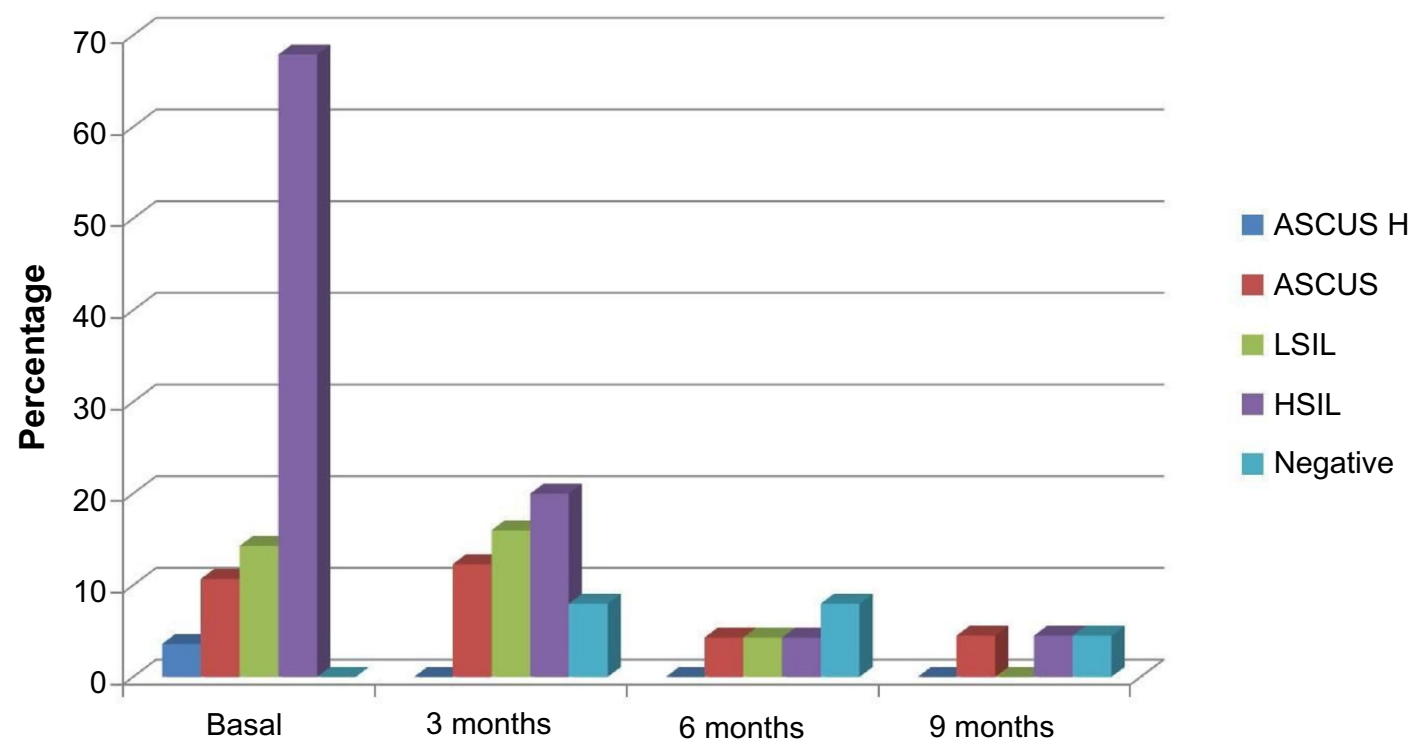

Figure 4 Cytological monitoring: different cytological results during the follow-up.

Abbreviations: ASCUS, atypical squamous cells of undetermined significance; ASCUS H, atypical squamous cells of undetermined significance high; HSIL, high-grade squamous intraepithelial lesion; LSIL, low-grade squamous intraepithelial lesion.

Table I Cytological monitoring: different cytological results during the follow-up

\begin{tabular}{lllll}
\hline$\%$ & Basal & At 3 months & At 6 months & At 9 months \\
\hline ASCUS H & $3.6 \%$ & - & - & - \\
ASCUS & $10.7 \%$ & $12.3 \%$ & $4.3 \%$ & $4.5 \%$ \\
LSIL & $14.3 \%$ & $16 \%$ & $4.3 \%$ & - \\
HSIL & $67.9 \%$ & $20 \%$ & $4.3 \%$ & $4.5 \%$ \\
Negative & $3.6 \%$ & $36 \%$ & $39 \%$ & $22.7 \%$ \\
Absent & - & $8 \%$ & $8 \%$ & $4.5 \%$ \\
\hline
\end{tabular}

Abbreviations: ASCUS, atypical squamous cells of undetermined significance; ASCUS H, atypical squamous cells of undetermined significance high; HSIL, high-grade squamous intraepithelial lesion; LSIL, low-grade squamous intraepithelial lesion.

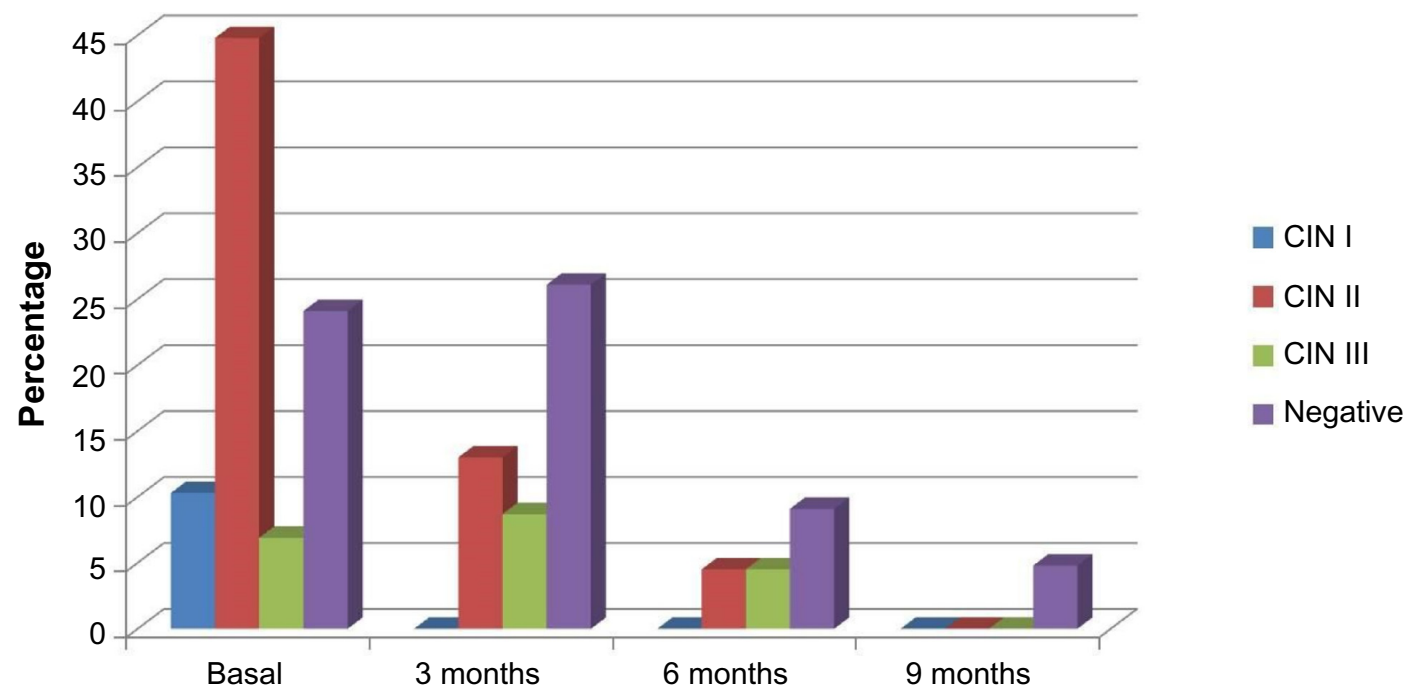

Figure 5 Histological monitoring: histological results during the follow-up. Abbreviation: $\mathrm{CIN}$, cervical intraepithelial neoplasm. 
Table 2 Histological monitoring: histological results during the follow-up

\begin{tabular}{lllll}
\hline$\%$ & Basal & At 3 months & At 6 months & At 9 months \\
\hline CIN I & $6.9 \%$ & - & - & - \\
CIN I,II & $3.4 \%$ & - & - & - \\
CIN II & $41.4 \%$ & $13 \%$ & $4.5 \%$ & - \\
CIN II,III & $6.9 \%$ & - & - & - \\
CIN III & - & $8.7 \%$ & $4.5 \%$ & - \\
Negative & $24.1 \%$ & $26.1 \%$ & $9.1 \%$ & $4.8 \%$ \\
\hline
\end{tabular}

Abbreviation: CIN, cervical intraepithelial neoplasm.

less than desirable. ${ }^{3}$ Of adolescents referred for colposcopy because of HSIL, only $50 \%$ have confirmed CIN II or III, suggesting that many HSIL cases are "overcalls." Up to $70 \%$ of these patients contracted the HPV virus at some point. Moscicki et al postulated the high prevalence of HPV in this age group as well as the ability to achieve spontaneous regression of the lesions. ${ }^{2,3}$

In 2007, Moore et $\mathrm{al}^{8}$ presented a prospective observational cohort study that included 501 patients. Thirty five percent had CIN II or higher, and $29 \%$ of patients with CIN II opted for watchful waiting; 18 months later, $65 \%$ showed regression.

There is scientific evidence that excisional treatment of high-grade lesions is closely related to the appearance of adverse reproductive events. In 2000, Sadler et $\mathrm{al}^{9}$ compared $^{2}$ cases of preterm delivery and its subtypes among a group of 426 women who opted for watchful waiting for colposcopic lesions compared with 652 who underwent conization, resulting in an increase in premature rupture of membranes before 37 weeks in the second group (relative risk: 2.7, 95\% confidence interval: 1.3-5.6).
Spanish protocols advise excisional treatment for all dysplasia of CIN II and higher. However, the American consensus in 2006 for the treatment of women with CIN suggests a follow-up colposcopy and cytology every 6 months, indicating that colposcopy is an alternative to conization in adolescent patients with histologicallyconfirmed CIN II or III. The same guide specifies that excisional treatment for CIN III is preferred. The reason is that HPV detected in adolescents with or without abnormal cytology at any one time point is likely to reflect a transient infection. ${ }^{10}$ Decreasing the number of conizations decreases the risk of preterm delivery, low birth weight, and perinatal mortality. Taking these facts into consideration, the option for conservative management of high-grade lesions in adolescents and young women is an alternative to surgery, but this is not yet included in many published clinical guidelines for the management of cervical dysplasia.

Our results suggest, as indicated by other authors, that conservative management of adolescent girls with high-grade cytological results is a good therapeutic option based on the latest knowledge about the natural history of HPV infection.

Table 3 Microbiological monitoring: percentage of low and high oncogenic risk HPV during the follow-up period

\begin{tabular}{|c|c|c|c|c|}
\hline & Basal & At 3 months & At 6 months & At 9 months \\
\hline \multicolumn{5}{|l|}{ High-risk serotype } \\
\hline One & $34.5 \%$ & $36 \%$ & $11.5 \%$ & $11.5 \%$ \\
\hline Various & $31 \%$ & $20 \%$ & $7.7 \%$ & $3.8 \%$ \\
\hline Without HR & $17.2 \%$ & $16 \%$ & $15.4 \%$ & $7.7 \%$ \\
\hline Not classified & $17.2 \%$ & - & $11.5 \%$ & $3.8 \%$ \\
\hline Not determined & - & $20 \%$ & $53.8 \%$ & $73.1 \%$ \\
\hline \multicolumn{5}{|l|}{ Low-risk serotype } \\
\hline One & $10.3 \%$ & $26 \%$ & $7.7 \%$ & $3.8 \%$ \\
\hline Various & $6.9 \%$ & $12 \%$ & - & - \\
\hline Without LR & $82.8 \%$ & $48 \%$ & $42.3 \%$ & $23.1 \%$ \\
\hline Not classified & - & - & - & - \\
\hline Not determined & - & $16 \%$ & $50 \%$ & $73.1 \%$ \\
\hline
\end{tabular}

Abbreviations: HPV, human papilloma virus; HR, high risk; LR, low risk. 


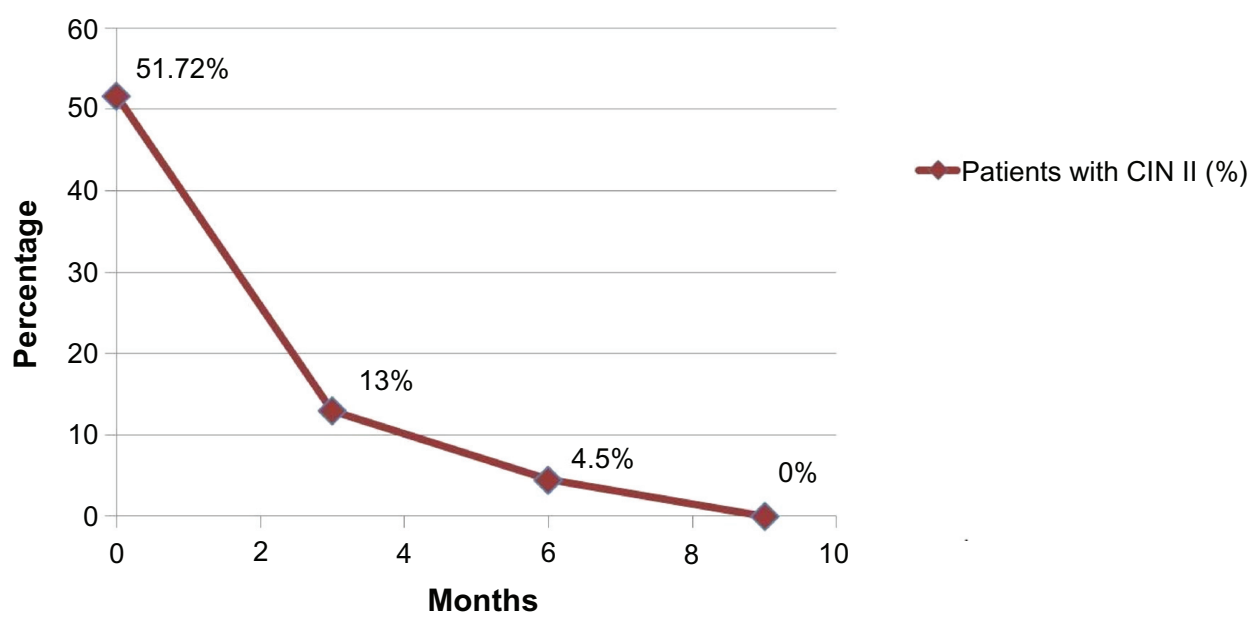

Figure 6 Evaluation of the CIN II during the study.

Abbreviation: CIN II, cervical intraepithelial neoplasm.

\section{Acknowledgments}

The authors would like to thank the following: the staff at the Cervical Pathology Unit of Obstetrics and Gynecology, Hospital 12 de Octubre, Fotunata Ortega Romanillos and Ana Martín, the nurses who worked on the study, the many others who facilitated the study, and most importantly, the women who participated in it. This study was not funded by any public or private institution.

\section{Disclosure}

The authors report no conflicts of interests in this work.

\section{References}

1. Burchell AN, Winer RL, de Sanjosé S, Franco EL. Chapter 6: Epidemiology and transmission dynamics of genital HPV infection. Vaccine. 2006;24(Suppl 3):S3/52-S3/61.

2. Moscicki AB, Shiboski S, Broering J, et al. The natural history of human papillomavirus infection as measured by repeated DNA testing in adolescent and young women. J Pediatr. 1998;132(2):277-284.

3. Moscicki AB, Shiboski S, Hills NK, et al. Regression of lowgrade squamous intra-epithelial lesions in young women. Lancet. 2004;364(9446):1678-1683.
4. American College of Obstetricians and Gynecologists. Guidelines for Women's Health Care. Washington, DC: The American College of Obstetricians and Gynecologists; 2002.

5. Kyrgiou M, Koliopoulos G, Martin-Hirsch P, Arbyn M, Prendiville W, Paraskevaidis E. Obstetric outcomes after conservative treatment for intraepithelial or early invasive cervical lesions: systematic review and meta-analysis. Lancet. 2006;367(9509):489-498.

6. Massad LS, Einstein MH, Huh WK, et al; 2012 ASCCP Consensus Guidelines Conference. 2012 updated consensus guidelines for the management of abnormal cervical cancer screening tests and cancer precursors. J Low Genit Tract Dis. 2013;17(5 Suppl 1):S1-S27.

7. Case AS, Rocconi RP, Straughn JM Jr, et al. Cervical intraepithelial neoplasia in adolescent women: incidence and treatment outcomes. Obstet Gynecol. 2006;108(6):1369-1374.

8. Moore K, Cofer A, Elliot L, Lanneau G, Walker J, Gold MA. Adolescent cervical dysplasia: histologic evaluation, treatment, and outcomes. Am J Obstet Gynecol. 2007;197(2):141.e1-141.e6.

9. Sadler L, Saftlas A, Wang W, Exeter M, Whittaker J, McCowan L. Treatment for cervical intraepithelial neoplasia and risk of preterm delivery. JAMA. 2004;291(17):2100-2106.

10. Boardman LA, Stanko C, Weitzen S, Sung CJ. Atypical squamous cells of undetermined significance: human papillomavirus testing in adolescents. Obstet Gynecol. 2005;105(4):741-746.
OncoTargets and Therapy

\section{Publish your work in this journal}

OncoTargets and Therapy is an international, peer-reviewed, open access journal focusing on the pathological basis of all cancers, potential targets for therapy and treatment protocols employed to improve the management of cancer patients. The journal also focuses on the impact of management programs and new therapeutic agents and protocols on

\section{Dovepress}

patient perspectives such as quality of life, adherence and satisfaction. The manuscript management system is completely online and includes a very quick and fair peer-review system, which is all easy to use. Visit http://www.dovepress.com/testimonials.php to read real quotes from published authors. 